Irish Math. Soc. Bulletin

Number 72, Winter 2013, 79-83

ISSN 0791-5578

\title{
OVER-CONSTRAINED TRIANGLES
}

\author{
FINBARR HOLLAND
}

\begin{abstract}
Inspired by a question that appeared on one Mathematics paper in the Irish Leaving Certificate Examination of 2013, we give a description of those triangles the measures of whose angles are rational multiples of $\pi$, and the squares of two of whose side lengths are rational numbers.
\end{abstract}

\section{INTRODUCTION}

As my colleague Des MacHale often says, 'mathematical inspiration can come from the most surprising of sources'. Indeed it can. Consideration of the infamous Question 8 on the second Higher Level Mathematics paper in the Irish Leaving Certificate Examination of 2013 led me to ask;

What triangles have the properties that the measures of their angles are whole numbers of degrees, and two of their side lengths are natural numbers?

This note provides an answer to a more general question, which hinges on the result given in the next section, that may be of independent interest.

\section{A Key Lemma}

Lemma 2.1. Suppose $0<t<\pi$ and $t$ is a rational multiple of $\pi$. If $\cos ^{2} t$ is rational, then $\cos t \in\left\{0, \pm \frac{1}{2}, \pm \frac{1}{\sqrt{2}}, \pm \frac{\sqrt{3}}{2}\right\}$.

Proof. We'll exploit the fact that, by hypothesis, there is a positive integer $m$ such that $\sin m t=0$. But, if $n$ is a positive integer, and $x$ is a real number, then $\sin (n x)=\sin x U_{n-1}(\cos x)$, where $U_{n}$ is

2010 Mathematics Subject Classification. 00A05, 33B10.

Key words and phrases. Triangles, Chebyshev polynomials of the second kind, Gauss Lemma.

Received on 17-9-2013; revised 18-10-2013.

I extend my thanks to Des MacHale for drawing my attention to Niven's book, and to Des and the referee for some helpful remarks about the presentation of this note. 
the $n$th degree Chebyshev polynomial of the second kind [1]. As is well-known, it follows from the trigonometric identity $\sin (n+$ 1) $x+\sin (n-1) x=2 \cos x \sin (n x)$ that, for all complex numbers $z$, $U_{0}(z)=1, U_{1}(z)=2 z$, and

$$
U_{n+1}(z)+U_{n-1}(z)=2 z U_{n}(z), n=1,2, \ldots .
$$

Using this we derive an explicit expression for $U_{n}$, namely $U_{n}=W_{n}$, where

$$
W_{n}(z)=\sum(-1)^{k}\left(\begin{array}{c}
n-k \\
k
\end{array}\right)(2 z)^{n-2 k} .
$$

(Here, and hereafter, we use the convention that, whenever $n, k$ are integers, then $\left(\begin{array}{l}n \\ k\end{array}\right)$ is zero unless $0 \leq k \leq n$.) To verify this statement, note that

$$
\begin{aligned}
W_{n+1}(z) & =\sum(-1)^{k}\left(\begin{array}{c}
n+1-k \\
k
\end{array}\right)(2 z)^{n+1-2 k} \\
& =\sum(-1)^{k}\left[\left(\begin{array}{c}
n-k \\
k
\end{array}\right)+\left(\begin{array}{c}
n-k \\
k-1
\end{array}\right)\right](2 z)^{n+1-2 k} \\
& =2 z W_{n}(z)+\sum(-1)^{k+1}\left(\begin{array}{c}
n-1-k \\
k
\end{array}\right)(2 z)^{n-1-2 k} \\
& =2 z W_{n}(z)-W_{n-1}(z),
\end{aligned}
$$

whence $U_{n}$ and $W_{n}$ satisfy the same linear recurrence relations, and so must be equal. As a consequence, it's clear that $W_{n}$ is a monic polynomial of degree $n$ with integer coefficients.

Note, too, that

$$
W_{n}(z)=\left\{\begin{array}{cc}
\sum(-1)^{k}\left(\begin{array}{c}
2 p-k \\
k
\end{array}\right)\left(4 z^{2}\right)^{p-k}, & \text { if } n=2 p, \\
2 z \sum(-1)^{k}\left(\begin{array}{c}
2 p+1-k \\
k
\end{array}\right)\left(4 z^{2}\right)^{p-k}, & \text { if } n=2 p+1 .
\end{array}\right.
$$

Since $0=\sin m t=\sin t W_{m-1}(\cos t)$, and $\sin t \neq 0$, it follows that $\cos t$ is a root of $W_{m-1}$. Hence, depending on the parity of $m$, either $\cos t=0$, and/or $4 \cos ^{2} t$ is a root of a monic polynomial with integer coefficients. But, by hypothesis, $\cos ^{2} t$ is a rational number. Hence, if $\cos t \neq 0$, then $4 \cos ^{2} t$ is an integer, by the Gauss Lemma, in which case $4 \cos ^{2} t \in\{1,2,3\}$, whence the stated result follows.

The following result, which is also recorded in [2], is a simple consequence.

Corollary 2.2. If $0<t<\pi, t$ is a rational multiple of $\pi$ and $\cos t$ is rational, then $\cos t \in\left\{0, \pm \frac{1}{2}\right\}$. 


\section{Classes of COnstrained triangles}

In this section we only consider triangles with the properties that the measures of their angles are rational multiples of $\pi$, and the squares of at least two of their side lengths are rational numbers. Therefore, for ease of typing, we assume throughout that the angular measures of the triangles under consideration are rational multiples of $\pi$.

Theorem 3.1. Triangles having the properties that the squares of their side lengths are rational numbers are of four kinds: either the measure of each angle is $\pi / 3$, or the measure of two angles is $\pi / 6$, or the measures of the three angles comprise the set $\{\pi / 12, \pi / 6,3 \pi / 4\}$ or the measure of one angle is $\pi / 2$. If they are of the latter kind, their acute angles are either equal or one is twice the other.

Proof. To establish this, suppose that $A B C$ is a triangle with the desired properties. Then, with the usual notation, $a^{2}, b^{2}$, and $c^{2}$ are rational numbers, and, by the Cosine Rule, so are $\cos ^{2}(A), \cos ^{2}(B)$, and $\cos ^{2}(C)$. Hence, by the Lemma, it follows that each of $\cos A$, $\cos B$, and $\cos C$ is a member of the set $\left\{0, \pm \frac{1}{2}, \pm \frac{1}{\sqrt{2}}, \pm \frac{\sqrt{3}}{2}\right\}$. Clearly, at most one of them can be zero or negative. If one is zero, $A B C$ is right-angled, and its acute angles are either equal or one is twice the other. If all are positive, they are equal, since if $x, y, z \in$ $\{1 / 6,1 / 4,1 / 3\}$ and $x+y+z=1$, then $x=y=z=1 / 3$, so that $A B C$ is equilateral. Finally, if one is negative, it is the cosine of one angle, $A$, say, whose measure is either $3 \pi / 4$ or $2 \pi / 3$. If $|\angle A|=3 \pi / 4$, then the pair $(|\angle B|,|\angle C|)$ is either $(\pi / 6, \pi / 12)$ or $(\pi / 12, \pi / 6)$. If $|\angle A|=2 \pi / 3$, then $|\angle B|=|\angle C|=\pi / 6$. These exhaust all the possibilities.

Corollary 3.2. If the side lengths of a triangle are rational numbers, then the triangle is either equilateral or an obtuse isosceles triangle with the measure of its largest angle being $2 \pi / 3$.

Proof. This time, $\cos A, \cos B, \cos C \in\left\{0, \pm \frac{1}{2}\right\}$. Hence, either $|\angle A|=$ $|\angle B|=|\angle C|=\frac{\pi}{3}$, or one of $|\angle A|,|\angle B|,|\angle C|$ is equal to $2 \pi / 3$, and each of the others is equal to $\pi / 6$.

We proceed to characterise the family of triangles with the property that the squares of two of their sides are rational numbers. 
Theorem 3.3. Suppose $A B C$ is a triangle in which $b^{2}$ and $c^{2}$ are rational numbers. Then either $b=c$ or the triangle is similar to $a$ small number of elementary triangles.

Proof. By the Sine Rule, the quotient $\sin ^{2} B / \sin ^{2} C$ is a rational number $r$, say. Let $u=4 \sin ^{2} B, v=4 \sin ^{2} C$, so that $u=r v$.

We distinguish two cases: (i) both $u, v$ are rational; (ii) both $u, v$ are irrational.

In case (i), it follows from Lemma 1 that $4-u, 4-v$ belong to the set $\{0,1,2,3\}$. In other words, $u, v \in\{1,2,3,4\}$. Hence, $\sin B, \sin C \in\{1 / 2,1 / \sqrt{2}, \sqrt{3} / 2,1\}$. From this it follows that either $|\angle B|=|\angle C|$, or one of $|\angle B|,|\angle C|$ is equal to either $3 \pi / 4$ or $2 \pi / 3$ and the other is equal to $\pi / 6$, or one of $|\angle B|,|\angle C|$ is equal to $\pi / 2$ and the other one belongs to the $\operatorname{set}\{\pi / 6, \pi / 4, \pi / 3\}$. Thus, in this case, $A B C$ is similar to one of five different triangles.

In case (ii), as follows from the proof of Lemma 1, $u, v$ are algebraic numbers, and so are roots of polynomials $f, g$ of least degree, whose coefficients are rational. Say $f(u)=g(v)=0$. Obviously, either (a) $\operatorname{deg} f \neq \operatorname{deg} g$ or (b) $\operatorname{deg} f=\operatorname{deg} g$. Suppose (a) happens, and, for definiteness, assume $\operatorname{deg} g<\operatorname{deg} f$. But then $u$ is a root of both $f$ and $t \rightarrow g(r t)$, a polynomial with rational coefficients of degree smaller than that of $f$, a contradiction. Hence, (a) is false. In case (b), $0=g(v)=f(r v)$, whence $0=r^{\operatorname{deg} f} g(v)-f(r v)=(r-1) h(v)$, say, where $h$ is a polynomial with rational coefficients, and $\operatorname{deg} h<$ $\operatorname{deg} g$. Hence, $r=1$, i.e., $u=v$. Equivalently, the angles $B, C$ are equal. This completes the description of $A B C$.

Finally, apropos our opening question, it should now be evident that triangles, with the property that two of their side lengths are unequal natural numbers, are right-angled ones in which one acute angle is twice the other.

\section{REFERENCES}

[1] P. Borwein, T. Erdélyi: Polynomials and Polynomial Inequalities, Graduate Texts in Mathematics, Springer-Verlag New York, Inc., 1995.

[2] I. Niven: Numbers: Rational and Irrational, New Mathematical Library, The Mathematical Association of America, 1961.

Finbarr Holland began teaching Mathematics in 1958, received his Ph.D. in Harmonic Analysis at University College, Cardiff in 1964, and is Professor Emeritus of Mathematics at University College Cork. Nowadays, he devotes much 
of his time composing and solving mathematical problems that appear in journals. As well, he follows the design and implementation of the syllabus and examination of Project Maths, a programme that fills him with foreboding about the teaching of Mathematics at second level in Ireland.

(F. Holland) School of Mathematical Sciences, University College Cork, Cork, Ireland.

E-mail address: f.holland@ucc.ie 